\title{
Certain maps preserving self-homotopy equivalences
}

\author{
Jin-ho Lee ${ }^{1}$ • Toshihiro Yamaguchi
}

Received: 10 November 2015 / Accepted: 18 September 2016 / Published online: 14 October 2016

(C) Tbilisi Centre for Mathematical Sciences 2016

\begin{abstract}
Let $\mathcal{E}(X)$ be the group of homotopy classes of self homotopy equivalences for a connected CW complex $X$. We consider two classes of maps, $\mathcal{E}$-maps and co$\mathcal{E}$-maps. They are defined as the maps $X \rightarrow Y$ that induce homomorphisms $\mathcal{E}(X) \rightarrow$ $\mathcal{E}(Y)$ and $\mathcal{E}(Y) \rightarrow \mathcal{E}(X)$, respectively. We give some rationalized examples related to spheres, Lie groups and homogeneous spaces by using Sullivan models. Furthermore, we introduce an $\mathcal{E}$-equivalence relation between rationalized spaces $X_{\mathbb{Q}}$ and $Y_{\mathbb{Q}}$ as a geometric realization of an isomorphism $\mathcal{E}\left(X_{\mathbb{Q}}\right) \cong \mathcal{E}\left(Y_{\mathbb{Q}}\right)$.
\end{abstract}

Keywords Self homotopy equivalence $\cdot \mathcal{E}$-Map $\cdot$ Co- $\mathcal{E}$-map $\cdot$ Rational homotopy Sullivan (minimal) model $\cdot$ Rational $\mathcal{E}$-map $\cdot$ Rational co- $\mathcal{E}$-map $\cdot$ Rationally $\mathcal{E}$-equivalent

Mathematics Subject Classification 55P62 - 55P10

\section{Introduction}

Needless to say, the based homotopy set $[X, Y]$ of based continuous maps from a based space $X$ to a based space $Y$ is a most interesting object in homotopy theory. In

Communicated by Jim Stasheff.

$凶$ Toshihiro Yamaguchi tyamag@kochi-u.ac.jp

Jin-ho Lee

sabforev@korea.ac.kr

1 Department of Mathematics, Korea University, Seoul, Korea

2 Faculty of Education, Kochi University, Kochi, Japan 
the following, all maps are based and we do not distinguish a homotopy class and the representative in a homotopy set. Let $X$ be a connected CW complex with base point $*$ and let

$$
\mathcal{E}(X)=\{[f] \in[X, X] \mid f: X \stackrel{\simeq}{\rightarrow} X\}
$$

be the group of homotopy classes of self-homotopy equivalences for $X$ with the operation given by the composition of homotopy classes. This group is important and has been closely studied as part of homotopy theory (for example, see [2,4-6,15-17]).

It is clear that $\mathcal{E}(X) \cong \mathcal{E}(Y)$ as a group if $X \simeq Y$. One of the difficulties of its computation or evaluation may be based on the fact that $\mathcal{E}(\quad)$ is not functorial, i.e., there is no suitable induced map between $\mathcal{E}(X)$ and $\mathcal{E}(Y)$ for the map $f: X \rightarrow Y$ in general. However, recall that, for example, the injection $i_{X}: X \rightarrow X \times Y$ and the projection $p_{Y}: X \times Y \rightarrow Y$ induce the natural monomorphisms $\mathcal{E}(X) \rightarrow \mathcal{E}(X \times Y)$ and $\mathcal{E}(Y) \rightarrow \mathcal{E}(X \times Y)$, respectively.

Definition 1.1 We say a map $f: X \rightarrow Y$ is an $\mathcal{E}$-map if there is a homomorphism $\phi_{f}: \mathcal{E}(X) \rightarrow \mathcal{E}(Y)$ such that

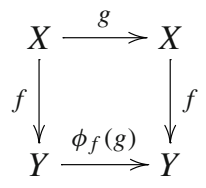

homotopically commutes for any element $g$ of $\mathcal{E}(X)$. We say the map $f: X \rightarrow Y$ is a co- $\mathcal{E}$-map if there is a homomorphism $\psi_{f}: \mathcal{E}(Y) \rightarrow \mathcal{E}(X)$ such that

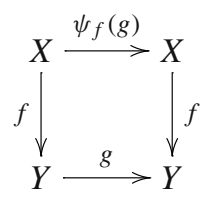

homotopically commutes for any element $g$ of $\mathcal{E}(Y)$.

Especially we consider the rationalized version of $\mathcal{E}$-maps and co- $\mathcal{E}$-maps by using Sullivan models $[8,9,18]$. Let $X_{\mathbb{Q}}$ be the rationalization of a nilpotent space $X[12]$.

Definition 1.2 A map $f: X \rightarrow Y$ between nilpotent spaces is a rational $\mathcal{E}$-map if the rationalization $f_{\mathbb{Q}}: X_{\mathbb{Q}} \rightarrow Y_{\mathbb{Q}}$ is an $\mathcal{E}$-map. Similarly a map $f: X \rightarrow Y$ between nilpotent spaces is a rational co- $\mathcal{E}$-map if the rationalization $f_{\mathbb{Q}}: X_{\mathbb{Q}} \rightarrow Y_{\mathbb{Q}}$ is a co- $\mathcal{E}$-map.

Question 1.3 When is a map a (rational) $\mathcal{E}$-map or a (rational) co- $\mathcal{E}$-map?

Theorem 1.4 Let $G$ be a compact connected Lie group and $H$ be a connected closed sub-Lie group of $G$. 
1. The inclusion $j: H \rightarrow G$ is a rational $\mathcal{E}$-map if $\pi_{*}(j) \otimes \mathbb{Q}$ is injective.

2. For the homogeneous space $G / H$, the projection map $f: G \rightarrow G / H$ is a rational co-E-map.

The assumption $\mathcal{E}(X) \cong \mathcal{E}(Y)$ does not, in general, imply $X$ and $Y$ are homotopy equivalent spaces. Finally, we consider the question: When is an isomorphism $\mathcal{E}\left(X_{\mathbb{Q}}\right) \cong \mathcal{E}\left(Y_{\mathbb{Q}}\right)$ realized as a composition of rational $\mathcal{E}$-maps and rational co-E-maps between $X_{\mathbb{Q}}$ and $Y_{\mathbb{Q}}$ ?

Definition 1.5 We say that nilpotent spaces $X$ and $Y$ are rationally $\mathcal{E}$-equivalent (denote as $X_{\mathbb{Q}} \underset{\mathcal{E}}{\sim} Y_{\mathbb{Q}}$ ) if there is a chain of spherically injective $\mathcal{E}$-maps and spherically injective co- $\mathcal{E}$-maps

$$
X_{\mathbb{Q}} \stackrel{f_{1}}{\rightarrow} Z_{1} \stackrel{f_{2}}{\leftarrow} \cdots \stackrel{f_{n}}{\leftarrow} Z_{n} \stackrel{f_{n+1}}{\rightarrow} Y_{\mathbb{Q}}
$$

( $Z_{i}$ are rational spaces) such that an isomorphism $\mathcal{E}\left(X_{\mathbb{Q}}\right) \cong \mathcal{E}\left(Y_{\mathbb{Q}}\right)$ is given by a composition of $n+1$-isomorphisms $\left\{\phi_{f_{i}}\right\}_{i}$ and $\left\{\psi_{f_{i}}\right\}_{i}$, i.e., $\phi_{f_{n+1}} \circ \psi_{f_{n}} \circ \cdots \circ \psi_{f_{2}} \circ \phi_{f_{1}}$ : $\mathcal{E}\left(X_{\mathbb{Q}}\right) \stackrel{\cong}{\rightrightarrows} \mathcal{E}\left(Y_{\mathbb{Q}}\right)$ or $\psi_{f_{1}} \circ \phi_{f_{2}} \circ \cdots \circ \phi_{f_{n}} \circ \psi_{f_{n+1}}: \mathcal{E}\left(Y_{\mathbb{Q}}\right) \stackrel{\cong}{\rightrightarrows} \mathcal{E}\left(X_{\mathbb{Q}}\right)$.

Remark 1.6 In this paper, we say that a map $f: X \rightarrow Y$ is spherically injective when $f_{\sharp}(u) \neq 0 \in \pi_{*}(Y)$ if $\operatorname{hur}_{X}(u) \neq 0$ for $u \in \pi_{*}(X)$. Here $h u r_{X}: \pi_{*}(X) \rightarrow H_{*}(X)$ is the Hurewicz homomorphism for a space $X$. Thus we have

(weakly) homotopy equivalent $\Rightarrow$ spherically injective $\Rightarrow$ homotopy non-trivial

If we admit the homotopy trivial maps as $f_{i}$, any isomorphism $\mathcal{E}\left(X_{\mathbb{Q}}\right) \cong \mathcal{E}\left(Y_{\mathbb{Q}}\right)$ induces $X_{\mathbb{Q}} \underset{\mathcal{E}}{\sim} Y_{\mathbb{Q}}$ by the constant map $*: X_{\mathbb{Q}} \rightarrow Y_{\mathbb{Q}}$.

Theorem 1.7 1. If $X$ and $Y$ are rationally homotopy equivalent, i.e., $X_{\mathbb{Q}} \simeq Y_{\mathbb{Q}}$, then $X_{\mathbb{Q}} \underset{\mathcal{E}}{\sim} Y_{\mathbb{Q}}$

2. For any $n, S_{\mathbb{Q}}^{2} \underset{\mathcal{E}}{\sim} \mathbb{C} P_{\mathbb{Q}}^{n}$ and $S_{\mathbb{Q}}^{4} \underset{\mathcal{E}}{\sim} \mathbb{H} P_{\mathbb{Q}}^{n}$.

3. When $m$ is even and $n$ is odd, $\left(S^{m} \vee S^{n}\right)_{\mathbb{Q}} \underset{\mathcal{E}}{\sim}\left(S^{m} \times S^{n}\right)_{\mathbb{Q}}$ if and only if $m \neq n+1$.

4. For odd-integers $1<m \leq n,\left(S^{m} \times S^{n}\right)_{\mathbb{Q}} \underset{\mathcal{E}}{\sim} E_{\mathbb{Q}} \underset{\mathcal{E}}{\sim} E_{\mathbb{Q}}^{\prime}$ for non-trivial fibrations $S^{m+n-1} \rightarrow E \stackrel{p}{\rightarrow} S^{m} \times S^{n}$ and $S^{2 m+n-2} \rightarrow E^{\prime} \stackrel{p^{\prime}}{\rightarrow} E$.

5. There are integers $m \neq n$ such that $S_{\mathbb{Q}}^{m} \underset{\mathcal{E}}{\sim} S_{\mathbb{Q}}^{n}$. For example, $S_{\mathbb{Q}}^{53} \underset{\mathcal{E}}{\sim} S_{\mathbb{Q}}^{67}$.

Remark 1.8 The proof of Theorem 1.7 (5) requires a rigid rational space $X$ of [3], which induces $\mathcal{E}(X)=\left\{i d_{X}\right\}$. For the total space $Z_{1}$ of a fibration

$$
X \rightarrow Z_{1} \rightarrow S_{\mathbb{Q}}^{m} \times S_{\mathbb{Q}}^{n},
$$

it is given by the sequence $S_{\mathbb{Q}}^{m} \stackrel{f_{1}}{\rightarrow} Z_{1} \stackrel{f_{2}}{\leftarrow} S_{\mathbb{Q}}^{n}$ of an $\mathcal{E}$-map $f_{1}$ with $\phi_{f_{1}}: \mathcal{E}\left(S_{\mathbb{Q}}^{m}\right) \cong$ $\mathcal{E}\left(Z_{1}\right)$ and a co- $\mathcal{E}$-map $f_{2}$ with $\psi_{f_{2}}: \mathcal{E}\left(Z_{1}\right) \cong \mathcal{E}\left(S_{\mathbb{Q}}^{n}\right)$. Because of the demand that 
$\mathcal{E}\left(Z_{1}\right) \cong \mathbb{Q}^{*}\left(\mathbb{Q}^{*}:=\mathbb{Q}-0\right)$, we need suitable restrictions about the pair $(m, n)$ and see that $(53,67)$ satisfies them in the proof. Of course, it depends on the model structure of $X$. So we may require more various types of rigid models for the proof of (5) in many cases of $(m, n)$. On the other hand, the authors cannot find an example that $S_{\mathbb{Q}}^{m} \underset{\mathcal{E}}{\nsim} S_{\mathbb{Q}}^{n}$ for some $(m, n)$.

Problem 1.9 If $\mathcal{E}(X) \cong \mathcal{E}(Y)$ for rational spaces $X$ and $Y$, does it hold that $X \underset{\mathcal{E}}{\sim} Y$ ?

Remark 1.10 For rational spaces $X, Y$ and $Z$, even if $Y \underset{\mathcal{E}}{\sim} Z$, it may not hold that $X \times Y \underset{\mathcal{E}}{\sim} X \times Z$. For example, when $X=S^{5}, Y=S^{2}$ and $Z=\mathbb{C} P^{2}, \mathcal{E}\left((X \times Y)_{\mathbb{Q}}\right) \cong$ $\mathbb{Q}^{*} \times \mathbb{Q}^{*}$ but $\mathcal{E}\left((X \times Z)_{\mathbb{Q}}\right)$ is isomorphic to the subgroup of lower triangular matrixes of $G L(2, \mathbb{Q})$.

In Sect. 2, we demonstrate the basic properties and provide examples in ordinary homotopy theory of $\mathcal{E}$-maps and co- $\mathcal{E}$-maps. In Sect. 3, we give some computations in rational homotopy theory using Sullivan minimal models.

\section{Some properties}

Recall that $[X$,$] is the covariant functor from the category of spaces to the category$ of sets, wherse for a map $f: Y \rightarrow Z$, the map $f_{*}(g):[X, Y] \rightarrow[X, Z]$ is given by $f_{*}(g)=f \circ g$. On the other hand, $[, Z]$ is the cotravariant functor. For the map $f: X \rightarrow Y$, the map $f^{*}(g):[Y, Z] \rightarrow[X, Z]$ is given by $f^{*}(g)=g \circ f$. The following lemma holds from $\phi_{f}(g) \circ f=f \circ g$ and $f \circ \psi_{f}(g)=g \circ f$.

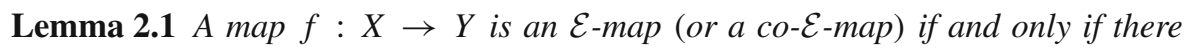
is a group homomorphism $\phi_{f}: \mathcal{E}(X) \rightarrow \mathcal{E}(Y)\left(\right.$ or $\left.\psi_{f}: \mathcal{E}(Y) \rightarrow \mathcal{E}(X)\right)$ where the following diagrams
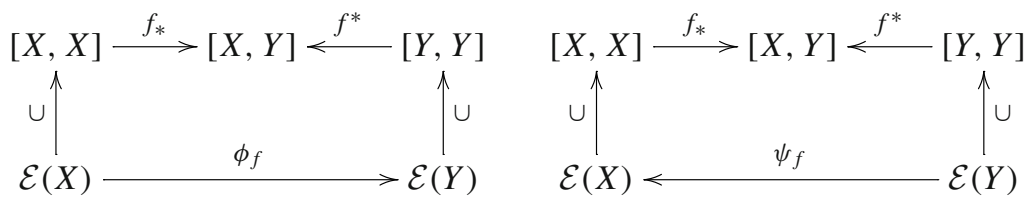

are commutative.

Of course, the maps $\phi_{f}$ and $\psi_{f}$ may not be uniquely determined for a map $f$.

Lemma 2.2 1. Ifmaps $f: X \rightarrow Y$ and $g: Y \rightarrow Z$ are $\mathcal{E}$-maps, then $g \circ f: X \rightarrow Z$ is an $\mathcal{E}$-map.

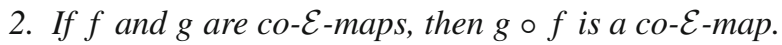

3. The constant map is both an $\mathcal{E}$-map and a co-E-map.

4. A homotopy equivalence map is both an $\mathcal{E}$-map and a co-E-map.

Proof 1. $\phi_{g \circ f}(h):=\phi_{g} \circ \phi_{f}(h)$ for $h \in \mathcal{E}(X)$. 
2. $\psi_{g \circ f}(h):=\psi_{f} \circ \psi_{g}(h)$ for $h \in \mathcal{E}(Z)$.

3. It is sufficient to put $\phi_{f}=\psi_{f}=*$, i.e., $\phi_{f}(g)=i d_{Y}$ and $\psi_{f}(g)=i d_{X}$ for any $g$.

4. It is sufficient to put $\phi_{f}(h):=f \circ h \circ f^{-1}$ for $h \in \mathcal{E}(X)$ and $\psi_{f}(h):=f^{-1} \circ h \circ f$ for $h \in \mathcal{E}(Y)$, where $f^{-1}$ is the homotopy inverse of $f$.

Definition 2.3 [11, Chapter 3] ([13]) Let $\alpha: X \rightarrow Y$ and $\beta: Z \rightarrow W$ be maps. $\Pi(\alpha, \beta)$ is the set of all homotopy classes of pairs $\left[f_{1}, f_{2}\right]$ such that

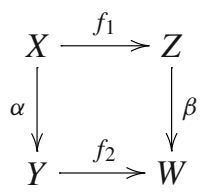

is homotopy commutative. Here a homotopy of $\left(f_{1}, f_{2}\right)$ is just a pair of homotopies $\left(f_{1 t}, f_{2 t}\right)$ such that $\beta f_{1 t}=f_{2 t} \alpha$. If $\left[f_{1}, f_{2}\right]$ has a two sided inverse in $\Pi(\alpha, \beta)$, we call $\left[f_{1}, f_{2}\right]$ a homotopy equivalence. If $\alpha=\beta$, we call $\left[f_{1}, f_{2}\right]$ a self-homotopy equivalence and denote the set of all self-homotopy equivalences by $\mathcal{E}(\alpha)$.

Lemma 2.4 For a map $f: X \rightarrow Y$,

1. $f$ is an $\mathcal{E}$-map if and only if $h: \mathcal{E}(f) \rightarrow \mathcal{E}(X)$ given by $h\left[g_{1}, g_{2}\right]=\left[g_{1}\right]$ is an epimorphism with a section.

2. $f$ is a co-E-map if and only if $h^{\prime}: \mathcal{E}(f) \rightarrow \mathcal{E}(Y)$ given by $h^{\prime}\left[g_{1}, g_{2}\right]=\left[g_{2}\right]$ is an epimorphism with a section.

Proof 1. Suppose that $f$ is an $\mathcal{E}$-map. Then we have a map $\phi_{f}: \mathcal{E}(X) \rightarrow \mathcal{E}(Y)$ such that $\phi_{f}(g) \circ f \simeq f \circ g$ for any $g \in \mathcal{E}(X)$. Thus we have $\left[g, \phi_{f}(g)\right] \in \mathcal{E}(f)$ and $h\left[g, \phi_{f}(g)\right]=[g]$ and $h$ is epimorphic. Next suppose that $h$ is an epimorphism. For any $[g] \in \mathcal{E}(X)$, we have $\left[g^{\prime}, g^{\prime \prime}\right] \in \mathcal{E}(f)$ such that $h\left[g^{\prime}, g^{\prime \prime}\right]=[g]$. So $g$ is homotopic to $g^{\prime}$. Since $\left[g^{\prime}, g^{\prime \prime}\right] \in \mathcal{E}(f), g^{\prime}$ and $g^{\prime \prime}$ are homotopy equivalences and $g^{\prime \prime} \circ f \simeq f \circ g^{\prime}$. Thus we can define a map $\phi_{f}: \mathcal{E}(X) \rightarrow \mathcal{E}(Y)$ by $\phi_{f}(g)=\pi \circ s[g]$ where $\pi: \mathcal{E}(f) \rightarrow \mathcal{E}(Y)$ is the natural projection and $s$ is the section of the assumption. Hence, $f$ is an $\mathcal{E}$-map.

2. Suppose that $f$ is a co- $\mathcal{E}$-map. Then we have a map $\psi_{f}: \mathcal{E}(Y) \rightarrow \mathcal{E}(X)$ such that $g \circ f \simeq f \circ \psi_{f}(g)$ for any $g \in \mathcal{E}(Y)$. So we have $\left[\psi_{f}(g), g\right] \in \mathcal{E}(f)$ and $h^{\prime}\left[\psi_{f}(g), g\right]=[g]$. Thus $h^{\prime}$ is epimorphic. Next suppose that $h^{\prime}$ is an epimorphism. For any $[g] \in \mathcal{E}(Y)$, we have $\left[g^{\prime}, g^{\prime \prime}\right] \in \mathcal{E}(f)$ such that $h\left[g^{\prime}, g^{\prime \prime}\right]=[g]$ and thus $g$ is homotopic to $g^{\prime \prime}$. Since $\left[g^{\prime}, g^{\prime \prime}\right] \in \mathcal{E}(f), g^{\prime}$ and $g^{\prime \prime}$ are homotopy equivalences and $g^{\prime \prime} \circ f \simeq f \circ g^{\prime}$. Then we can define a map $\psi_{f}: \mathcal{E}(Y) \rightarrow \mathcal{E}(X)$ by $\psi_{f}(g)=h \circ s^{\prime}[g]$ for the section $s^{\prime}$. Hence, $f$ is a co- $\mathcal{E}$-map.

Theorem 2.5 Let $\eta: S^{3} \rightarrow S^{2}$ and $v: S^{7} \rightarrow S^{4}$ be the Hopf fibrations with fibre $S^{1}$ and $S^{3}$, respectively. Let $\epsilon_{3}: S^{11} \rightarrow S^{3}$ be the generator of $\pi_{11}\left(S^{3}\right) \cong \mathbb{Z}_{2}$ [19]. Then 
1. $\eta$ is a co-E-map, but not an $\mathcal{E}$-map,

2. $v$ is neither an $\mathcal{E}$-map nor a co-E-map and

3. $\epsilon_{3}$ is both an $\mathcal{E}$-map and a co-E-map.

Proof 1. From [14, Example 4.2 (i)], we have $\Pi(\eta, \eta)=\left\{\left(k^{2} \iota_{3}, k \iota_{2}\right) \mid k \in \mathbb{Z}\right\}$ as a set. Therefore, we have a homotopy commutative diagram

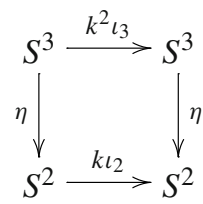

It is well known that $\mathcal{E}\left(S^{n}\right)=\left\{\iota_{n},-\iota_{n}\right\} \cong \mathbb{Z}_{2}$. Since $\left(\iota_{3},-\iota_{2}\right),\left(\iota_{3}, \iota_{2}\right) \in \Pi(\eta, \eta)$, $\eta$ is a co- $\mathcal{E}$-map. However, there is no map $f: S^{2} \rightarrow S^{2}$ such that $\left(-\iota_{3}, f\right) \in$ $\Pi(\eta, \eta)$. Thus $\eta$ is not an $\mathcal{E}$-map.

2. From [14, Example 4.2 (ii)], we have $\Pi(v, v)=\left\{\left(k^{2} \iota_{7}, k \iota_{4}\right) \mid k(k-1) \equiv\right.$ $0(\bmod 8)\}$ as a set. Therefore, we have a homotopy commutative diagram

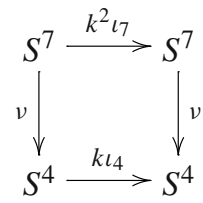

Since there are no maps $f: S^{7} \rightarrow S^{7}$ and $g: S^{4} \rightarrow S^{4}$ such that $\left(f,-\iota_{4}\right),\left(-\iota_{7}, g\right) \in \Pi(v, v), v$ is neither an $\mathcal{E}$-map nor a co- $\mathcal{E}$-map.

3. From [14, Example 4.2 (iv)], we have $\Pi\left(\epsilon_{3}, \epsilon_{3}\right)=\left\{\left((d+2 s) \iota_{11}, d \iota_{3}\right) \mid d, s \in\right.$ $\mathbb{Z}\} \cong \mathbb{Z} \times \mathbb{Z}$ as a group. Therefore we have a homotopy commutative diagram

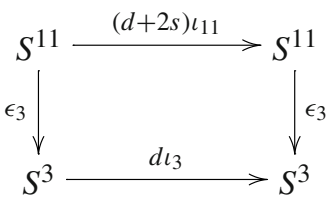

Since $\left(\iota_{11}, \iota_{3}\right),\left(-\iota_{11},-\iota_{3}\right) \in \Pi\left(\varepsilon_{3}, \varepsilon_{3}\right), \varepsilon_{3}$ is both an $\mathcal{E}$-map and a co- $\mathcal{E}$-map.

Example 2.6 1. Let $e: X \rightarrow \Omega \Sigma X$ be the adjoint of $i d_{\Sigma X}$ from the one-to-one correspondence $[X, \Omega \Sigma X] \cong[\Sigma X, \Sigma X]$. We know that $e(x)(t)=\langle x, t\rangle$. Let $f$ be a self homotopy equivalence on $X$, that is, $f \in \mathcal{E}(X)$ and let $f^{\prime}$ be a homotopy inverse of $f$. It is clear that the map $\Sigma f: \Sigma X \rightarrow \Sigma X, \Sigma f\langle x, t\rangle=\langle f(x), t\rangle$, is a homotopy equivalence with homotopy inverse $\Sigma f^{\prime}$. Then we define a map $\widetilde{f}: \Omega \Sigma X \rightarrow \Omega \Sigma X$ by $\widetilde{f}(\alpha)(t)=\Sigma f(\alpha(t))$. Define another map $\tilde{f}^{\prime}: \Omega \Sigma X \rightarrow$ $\Omega \Sigma X$ by $\tilde{f}^{\prime}(\alpha)(t)=\Sigma f^{\prime}(\alpha(t))$. Clearly we have $\widetilde{f} \circ \widetilde{f}^{\prime} \simeq i d$ and $\tilde{f}^{\prime} \circ \widetilde{f} \simeq i d$. Moreover we have $e(f(x))(t)=\langle f(x), t\rangle$ and $\tilde{f}(e(x))(t)=\Sigma f(e(x)(t))=$ 
$\Sigma f\langle x, t\rangle=\langle f(x), t\rangle$. Therefore we have a commutative diagram

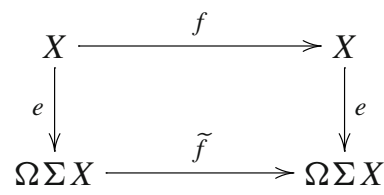

Thus $e: X \rightarrow \Omega \Sigma X$ is an $\mathcal{E}$-map.

2. Let $\pi: \Sigma \Omega Y \rightarrow Y$ be the adjoint of $i d_{\Omega Y}$ from the one-to-one correspondence $[\Sigma \Omega Y, Y] \cong[\Omega Y, \Omega Y]$. We know that $\pi\langle\alpha, t\rangle=\alpha(t)$. Let $g$ be a self homotopy equivalence on $Y$, that is $g \in \mathcal{E}(Y)$ and let $g^{\prime}$ be a homotopy inverse of $g$. Then we define a map $\widetilde{g}: \Sigma \Omega Y \rightarrow \Sigma \Omega Y$ by $\widetilde{g}\langle\alpha, t\rangle=\langle g \circ \alpha, t\rangle$ and $\widetilde{g^{\prime}}: \Sigma \Omega Y \rightarrow \Sigma \Omega Y$ by $\widetilde{g^{\prime}}\langle\alpha, t\rangle=\left\langle g^{\prime} \circ \alpha, t\right\rangle$. Clearly we have $\widetilde{g} \circ \widetilde{g^{\prime}} \simeq i d$ and $\widetilde{g^{\prime}} \circ \widetilde{g} \simeq i d$. Moreover we have $(\pi \circ \widetilde{g})\langle\alpha, t\rangle=\pi\langle g \circ \alpha, t\rangle=(g \circ \alpha)(t)$ and $(g \circ \pi)\langle\alpha, t\rangle=g(\alpha(t))$. Therefore we have a commutative diagram

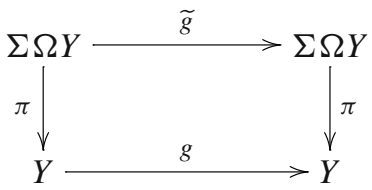

Therefore $\pi: \Sigma \Omega Y \rightarrow Y$ is a co- $\mathcal{E}$-map.

Example 2.7 There is a natural homomorphism $\mathcal{E}\left(X_{(n)}\right) \rightarrow \mathcal{E}\left(X_{(n-1)}\right)$ obtained by restricting the map to a lower Postnikov section [2, p. 27]. Thus the principal $K\left(\pi_{n}(X), n\right)$-fibration $X_{(n)} \rightarrow X_{(n-1)}$ is an $\mathcal{E}$-map. The restriction map $X \rightarrow X_{(n-1)}$ is also an $\mathcal{E}$-map. On the other hand, for the $n$-skeleton $X^{(n)}$, the inclusions $X^{(n)} \rightarrow$ $X^{(n+1)}$ and $X^{(n)} \rightarrow X$ are both co- $\mathcal{E}$-maps.

Remark 2.8 Recall that a space $X$ is said to be (homotopically) rigid when $\mathcal{E}(X)=$ $\left\{i d_{X}\right\}[3,7]$. If $X$ is rigid, then every map $f: X \rightarrow Y$ is an $\mathcal{E}$-map by $\phi_{f}\left(i d_{X}\right)=i d_{Y}$. If $Y$ is rigid, then every map $f: X \rightarrow Y$ is a co- $\mathcal{E}$-map by $\psi_{f}\left(i d_{Y}\right)=i d_{X}$. Also we can construct infinitely many examples of $\mathcal{E}$-maps and co- $\mathcal{E}$-maps by using the functor of [7, Remark 2.8].

\section{Computations in Sullivan models}

We assume that $X$ is a nilpotent CW complex. Let $M(X)=(\Lambda V, d)$ be the Sullivan minimal model of $X$ [18]. It is a free $\mathbb{Q}$-commutative differential graded algebra (DGA) with a $\mathbb{Q}$-graded vector space $V=\bigoplus_{i \geq 1} V^{i}$ where $\operatorname{dim} V^{i}<\infty$ and a decomposable differential; i.e., $d\left(V^{i}\right) \subset\left(\Lambda^{+} V \cdot \Lambda^{+} V\right)^{i+1}$ and $d \circ d=0$. Here $\Lambda^{+} V$ is the ideal of $\Lambda V$ generated by elements of positive degree. The degree of a homogeneous element $x$ of a graded algebra is denoted as $|x|$. Then $x y=(-1)^{|x||y|} y x$ and $d(x y)=d(x) y+(-1)^{|x|} x d(y)$. Note that $M(X)$ determines the rational homotopy 
type of $X$. In particular, $H^{*}(\Lambda V, d) \cong H^{*}(X ; \mathbb{Q})$ and $V^{i} \cong H \operatorname{Hom}\left(\pi_{i}(X), \mathbb{Q}\right)$. Refer to [8] for details.

Let Aut $M$ be the group of DGA-automorphisms of a DGA $M$. For a nilpotent space $X$ and a (not necessarily minimal) model $M(X)$, there is a group isomorphism

$$
\mathcal{E}\left(X_{\mathbb{Q}}\right) \cong \mathcal{E}(M(X))
$$

where $\mathcal{E}(M(X))=\operatorname{Aut} M(X) / \sim$ is the group of self-DGA-homotopy equivalence classes of $M(X)$ [18]. Now recall about "DGA-homotopy" in [9]: In general, two maps $f: M(Y) \rightarrow M(X)$ and $g: M(Y) \rightarrow M(X)$ are DGA-homotopic (denote as $f \sim g$ ) if there is a DGA-map $H: M(Y) \rightarrow M(X) \otimes \Lambda(t, d t)$ such that $\left.H\right|_{t=0, d t=0}=f$ and $\left.H\right|_{t=1, d t=0}=g$. Here $|t|=0$ and $|d t|=1$ with $d(t)=d t, d(d t)=0$.

The group $\mathcal{E}(M(X))$ does not depend on choosing a model of $X$. For example, the minimal model $M=M\left(S^{2 n+1}\right)=(\Lambda w, 0)$ and a non-minimal model $M^{\prime}=$ $(\Lambda(y, w, v), D)$ with $|y|=2,|w|=2 n+1,|v|=1, D y=0, D w=y^{n+1}$ and $D v=y$ are both models of $S^{2 n+1}$. Obviously we have $\mathcal{E}(M) \cong \mathbb{Q}^{*}$ by $w \rightarrow$ aw for $a \in \mathbb{Q}^{*}$. On the other hand, in $\mathcal{E}\left(M^{\prime}\right)$ we can set $H(y)=c y t+c v d t, H(v)=c v t$ and $H(w)=a w+b y^{n} v+b^{\prime} y^{n} v t^{n+1}$ with $a, b, b^{\prime}, c \in \mathbb{Q}^{*}$. Then $a+b=0$ and $b^{\prime}=c^{n+1}$ from $D \circ H=H \circ D$. Thus two maps $f \in \operatorname{Aut} M^{\prime}$ given by $f(y)=f(v)=0$, $f(w)=a\left(w-y^{n} v\right)$ and $g \in \operatorname{Aut} M^{\prime}$ given by $g(y)=c y, g(v)=c v, g(w)=$ $a\left(w-y^{n} v\right)+c^{n+1} y^{n} v$ are DGA-homotopic. Hence we have $\mathcal{E}\left(M^{\prime}\right)=\mathcal{E}(M) \cong \mathbb{Q}^{*}$ as in Example 3.3(1) below.

Remark 3.1 From the universality of the localization [12], the rationalization map $l: X \rightarrow X_{\mathbb{Q}}$ is an $\mathcal{E}$-map, but it is not a co- $\mathcal{E}$-map in general. For example, when $X=S^{3}$, the elements $f$ of $\mathcal{E}(M(X))=\mathcal{E}(\Lambda(x), 0)$ with $f(x)=a x$ for $a \neq \pm 1 \in$ $\mathbb{Q}^{*}=\mathbb{Q}-0$ can not be realized as a homotopy equivalence of $X$.

The model of a map $f: X \rightarrow Y$ between nilpotent spaces is given by a relative model:

$$
M(Y)=\left(\Lambda W, d_{Y}\right) \stackrel{i}{\rightarrow}(\Lambda W \otimes \Lambda V, D) \stackrel{q}{\rightarrow}(\Lambda V, \bar{D})
$$

with $\left.D\right|_{\Lambda W}=d_{Y}$ and the minimal model $(\Lambda V, \bar{D})$ of the homotopy fiber of $f$. It is well known that there is a quasi-isomorphism $M(X) \rightarrow(\Lambda W \otimes \Lambda V, D)$ [8]. Then Definition 1.2 is translated to

Definition 3.2 Let $f: X \rightarrow Y$ be a map between nilpotent spaces.

1. The map $f$ is a rational $\mathcal{E}$-map if and only if there is a homomorphism $\phi_{f}$ : $\mathcal{E}(\Lambda W \otimes \Lambda V, D) \rightarrow \mathcal{E}\left(\Lambda W, d_{Y}\right)$ such that

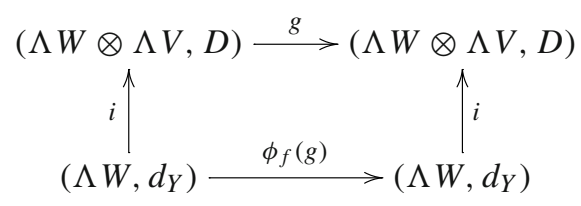

is DGA-homotopy commutative for any element $g$ of $\mathcal{E}(\Lambda W \otimes \Lambda V, D)$. 
2. The map $f$ is a rational co- $\mathcal{E}$-map if and only if there is a homomorphism $\psi_{f}$ : $\mathcal{E}\left(\Lambda W, d_{Y}\right) \rightarrow \mathcal{E}(\Lambda W \otimes \Lambda V, D)$ such that

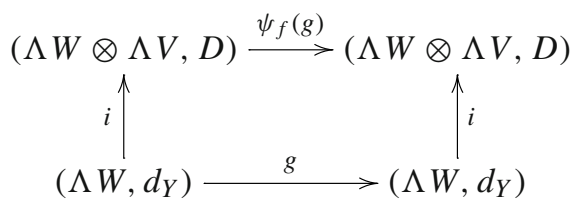

is DGA-homotopy commutative for any element $g$ of $\mathcal{E}\left(\Lambda W, d_{Y}\right)$.

Example 3.3 1. For the Hopf fibration $S^{1} \rightarrow S^{2 n+1} \stackrel{f}{\rightarrow} \mathbb{C} P^{n}$, the relative model is given by

$$
\left(\Lambda(y, w), d_{Y}\right) \rightarrow(\Lambda(y, w, v), D) \rightarrow(\Lambda(v), 0)
$$

with $|y|=2,|w|=2 n+1,|v|=1, d_{Y} w=y^{n+1}$ and $D v=y$. We can identify $\mathcal{E}\left(\mathbb{C} P_{\mathbb{Q}}^{n}\right)$ as $\mathbb{Q}^{*}$ by $g(y)=a y$ and $g(w)=a^{n+1} w$ for $g \in \mathcal{E}\left(\mathbb{C} P_{\mathbb{Q}}^{n}\right)$ and $a \in \mathbb{Q}^{*}$. Also we have $\mathcal{E}\left(S_{\mathbb{Q}}^{2 n+1}\right)=\mathcal{E}(\Lambda(y, w, v), D)=\mathcal{E}(\Lambda w, 0) \cong \mathbb{Q}^{*}$. Then there is a homomorphism

$$
\psi_{f}: \mathbb{Q}^{*} \cong \mathcal{E}\left(\mathbb{C} P_{\mathbb{Q}}^{n}\right) \rightarrow \mathcal{E}\left(S_{\mathbb{Q}}^{2 n+1}\right) \cong \mathbb{Q}^{*}
$$

which is given by $\psi_{f}(a)=a^{n+1}$ for $a \in \mathbb{Q}^{*}$. Thus $f$ is a rational co- $\mathcal{E}$-map, but it is not a rational $\mathcal{E}$-map.

2. Let $X$ be the pullback of the sphere bundle of the tangent bundle of $S^{m+n}$ by the canonical degree 1 map $S^{m} \times S^{n} \rightarrow S^{m+n}$ for odd integers $m$ and $n$. Then it is the total space of a fibration $S^{m+n-1} \rightarrow X \stackrel{f}{\rightarrow} S^{m} \times S^{n}$ whose model is

$$
\left(\Lambda\left(w_{1}, w_{2}\right), 0\right) \rightarrow\left(\Lambda\left(w_{1}, w_{2}, u\right), D\right) \rightarrow(\Lambda(u), 0)
$$

with $\left|w_{1}\right|=m,\left|w_{2}\right|=n,|u|=m+n-1$ and $D u=w_{1} w_{2}$ is both a rational $\mathcal{E}$-map and a rational co- $\mathcal{E}$-map.

3. The fibration $S^{m} \times S^{m+n-1} \rightarrow X \stackrel{f}{\rightarrow} S^{n}$ ( $m \neq n$ are odd) whose model is

$$
(\Lambda(w), 0) \rightarrow(\Lambda(w, v, u), D) \rightarrow(\Lambda(v, u), 0)
$$

where $|w|=n,|v|=m,|u|=m+n-1$ and $D u=w v$ with $m, n$ odd is both a rational $\mathcal{E}$-map and a rational co- $\mathcal{E}$-map.

4. For the fibration $\mathbb{C} P^{n-1} \rightarrow \mathbb{C} P^{2 n-1} \stackrel{f}{\rightarrow} S^{2 n}$ given by

$$
\left(\Lambda(y, w), d_{Y}\right) \rightarrow(\Lambda(y, w, x, v), D) \rightarrow(\Lambda(x, v), \bar{D})
$$


with $|y|=2 n,|w|=4 n-1,|x|=2,|v|=2 n-1, d_{Y} y=0, d_{Y} w=y^{2}$, $D x=\bar{D} x=0, D v=y-x^{n}$ and $\bar{D} v=x^{n}$, the map $f$ is a rational $\mathcal{E}$-map given by $\phi_{f}(a)=a^{n}$ for $a \in \mathbb{Q}^{*}$ but not a rational co- $\mathcal{E}$-map.

Example 3.4 For an $n$-dimensional manifold $X$, the collapsing map of lower cells $f: X \rightarrow S^{n}$ is an $\mathcal{E}$-map. Indeed, from the commutative diagram between cofibrations

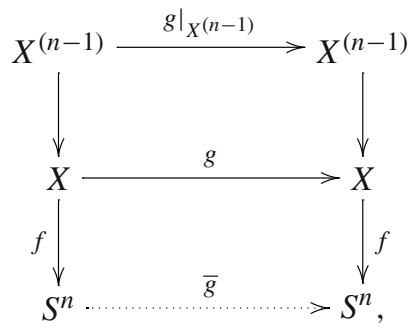

we have $\phi_{f}(g)=\bar{g}$, but it is not a (rational) co- $\mathcal{E}$-map in general. For example, the collapsing map of lower cells $f: X=\mathbb{C} P^{n} \rightarrow S^{2 n}=Y$ induces a DGA-map

$$
f^{*}: M(Y)=\left(\Lambda(y, w), d_{Y}\right) \rightarrow\left(\Lambda(x, v), d_{X}\right)=M(X)
$$

with $d_{Y} w=y^{2}, d_{X} v=x^{n+1}, f^{*}(y)=x^{n}$ and $f^{*}(w)=x^{n-1} v$. The map $f$ is a rational $\mathcal{E}$-map by $\phi_{f}(a)=a^{n}$ for $a \in \mathbb{Q}^{*}$ but not a rational co-E-E-map. Indeed, for $g^{*}(y)=$ ay with $a \notin\left(\mathbb{Q}^{*}\right)^{\times m}:=\mathbb{Q}^{*} \cdot \mathbb{Q}^{*} \cdots \mathbb{Q}^{*}$ (m-times), we cannot define $\psi_{f *}\left(g^{*}\right)$.

Example 3.5 Let $\Omega Y=\operatorname{map}\left(\left(S^{1}, *\right),(Y, *)\right)$ be the base point preserving the loop space of a simply connected space $Y$ and $L Y=\operatorname{map}\left(S^{1}, Y\right)$, the free loop space of $Y$. We consider the evaluation map $f: L Y \rightarrow Y$ with $f(\sigma)=\sigma(*)$. It is a co- $\mathcal{E}$-map by $\psi_{f}(g)(h)=g \circ h$ for $g \in \mathcal{E}(Y)$. What is the (rational) homotopical condition of $Y$ that allows $f$ to be a (rational) $\mathcal{E}$-map? According to [20], the relative model of the free loop fibration $\Omega Y \rightarrow L Y \stackrel{f}{\rightarrow} Y$ :

$$
M(Y)=(\Lambda V, d) \rightarrow(\Lambda V \otimes \Lambda \bar{V}, D) \rightarrow(\Lambda \bar{V}, 0)
$$

with $M(L Y)=(\Lambda V \otimes \Lambda \bar{V}, D)$ is defined as follow: The graded vector space $\bar{V}$ satisfies $\bar{V}^{n} \cong V^{n-1}$ for $n>0$ and denote by $s: V \rightarrow \bar{V}(s(v):=\bar{v})$ this isomorphism of degree -1 . There is a unique extension of $s$ into a derivation of algebra $s: \Lambda V \otimes$ $\Lambda \bar{V} \rightarrow \Lambda V \otimes \Lambda \bar{V}$ such that $s(\bar{V})=0$. The differential $D$ is given by $D(v)=d(v)$ for $v \in V$ and $D(\bar{v})=-s \circ d(v)$ for $\bar{v} \in \bar{V}$.

If every DGA-isomorphism $g$ of $(\Lambda V \otimes \Lambda \bar{V}, D)$ satisfies $\left.g\right|_{\Lambda V} \in \mathcal{E}(\Lambda V, d)$, then $f(M(f))$ is a rational $\mathcal{E}$-map by $\phi_{f}(g)=\left.g\right|_{\Lambda V}$.

1. When $Y=S^{n}$, we observe that the map $f$ is a rational $\mathcal{E}$-map. If $n$ is even, $M\left(S^{n}\right)=(\Lambda(x, y), d)$ with $|x|=n,|y|=2 n+1, d x=0$ and $d y=x^{2}$. For example, when $n=2$, note that there is no DGA-map $g(x)=x+\bar{y}$. 
2. When $Y=S^{m} \times S^{n}$ for odd integers $m<n$, the map $f$ is a rational $\mathcal{E}$-map if and only if $m-1$ is not a divisor of $n-1$. Indeed, let $M\left(S^{m} \times S^{n}\right)=(\Lambda(x, y), 0)$. When $n-1=a(m-1)$ for an integer $a>1$, there is a DGA-isomorphism $g:(\Lambda(x, y, \bar{x}, \bar{y}), 0) \rightarrow(\Lambda(x, y, \bar{x}, \bar{y}), 0)$ with $g(x)=x, g(\bar{x})=\bar{x}, g(\bar{y})=\bar{y}$ and $g(y)=y+\bar{x}^{a-1} x$. Then $f$ cannot be a rational $\mathcal{E}$-map. When $n-1 \neq a(m-1)$ for any $a$, a self-map $g$ is given by $g(x)=x$ and $g(y)=y$ from the degree reason.

Proof of Theorem 1.4. (1) Note that $\pi_{*}(j)_{\mathbb{Q}}$ is injective if and only if the model of $j: H \rightarrow G$ is given as the projection $M(G) \cong\left(\Lambda\left(v_{1}, \ldots, v_{k}, u_{1}, \ldots, u_{l}\right), 0\right) \rightarrow$ $\left(\Lambda\left(v_{1}, \ldots, v_{k}\right), 0\right) \cong M(H)$ after a suitable basis change. Then we can define as $\phi_{j}(g)=g \otimes 1_{\Lambda\left(u_{1}, \ldots, u_{l}\right)}$ for any $g \in \mathcal{E}\left(\Lambda\left(v_{1}, \ldots, v_{k}\right), 0\right)$.

For the $n$-dimensional unitary group $U(n), M(U(n))=M\left(S^{1} \times \cdots \times S^{2 n-1}\right)=$ $\left(\Lambda\left(v_{1}, \ldots, v_{n}\right), 0\right)$ with $\left|v_{i}\right|=2 i-1$. For the n-dimensional special unitary group $S U(n), M(S U(n))=\left(\Lambda\left(v_{1}, \ldots, v_{n-1}\right), 0\right)$ with $\left|v_{i}\right|=2 i+1$. For the n-dimensional symplectic group $\operatorname{Sp}(n), M(S p(n))=\left(\Lambda\left(v_{1}, \ldots, v_{n}\right), 0\right)$ with $\left|v_{i}\right|=4 i-1$.

Example 3.6 In general, for a connected closed sub-Lie group $H$ of a compact connected Lie group $G$, the inclusion $j: H \rightarrow G$, is not a rational $\mathcal{E}$-map. For example, the blockwise inclusion $j: S U(3) \times S U(3) \rightarrow S U(6)$ is not. Indeed, $M(S U(3) \times S U(3))=\left(\Lambda\left(u_{1}, w_{1}, u_{2}, w_{2}\right), 0\right)$ with $\left|u_{1}\right|=\left|w_{1}\right|=3\left|u_{2}\right|=\left|w_{2}\right|=5$ and $M(S U(6))=\left(\Lambda\left(v_{1}, v_{2}, v_{3}, v_{4}, v_{5}\right), 0\right)$ with $\left|v_{i}\right|=2 i+1 . M(j)\left(v_{i}\right)=u_{i}+w_{i}$ for $i=1,2$. Then we cannot define $\phi_{j}(g)$ for $g \in \mathcal{E}\left(\Lambda\left(u_{1}, w_{1}, u_{2}, w_{2}\right), 0\right)$ when $g\left(u_{i}\right)=u_{i} g\left(w_{i}\right)=-w_{i}$ for example.

Lemma 3.7 Let $X=S^{a_{1}} \times \cdots \times S^{a_{m}} \times Y$ and $Y=S^{b_{1}} \times \cdots \times S^{b_{n}}$ for odd-integers $a_{1} \leq \cdots \leq a_{m} \leq b_{1} \leq \cdots \leq b_{n}$. Then the second factor projection map $f: X \rightarrow Y$ is a rational $\mathcal{E}$-map if and only if there are no subsets $\left\{i_{1}, \ldots, i_{k}\right\}$ of $\{1, \ldots, m\}$ and $\left\{j_{1}, \ldots, j_{k}\right\}$ of $\{1, \ldots, n\}$ with $b_{k}=a_{i_{1}}+\cdots+a_{i_{k}}+b_{j_{1}}+\cdots+b_{j_{k}}$ for $k=1, \ldots, n$.

Proof Put $M(X)=\left(\Lambda\left(x_{1}, \ldots, x_{m}, y_{1}, \ldots, y_{n}\right), 0\right)$ and $M(Y)=\left(\Lambda\left(y_{1}, \ldots, y_{n}\right), 0\right)$ with $\left|x_{i}\right|=a_{i}$ and $\left|y_{i}\right|=b_{i}$. If $b_{k}=a_{i_{1}}+\cdots+b_{j_{k}}$, there is a map $g \in \mathcal{E}(M(X))$ such that

$$
g\left(x_{i}\right)=x_{i} \quad(i \leq m), \quad g\left(y_{i}\right)=y_{i} \quad(i \neq k), \quad g\left(y_{k}\right)=y_{k}+x_{i_{1}} \cdots x_{i_{k}} y_{j_{1}} \cdots y_{j_{k}}
$$

and $M(f)\left(y_{i}\right)=y_{i}$ for all $i$. Then we can not have a DGA-homotopy commutative diagram

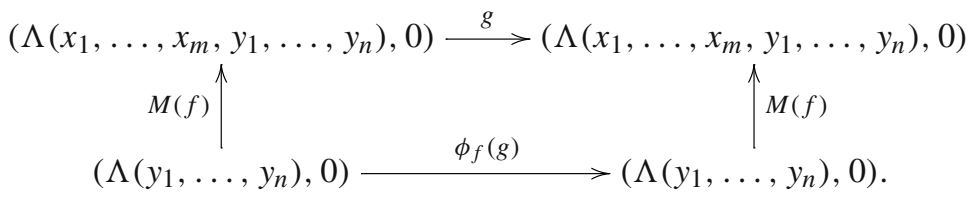

If $b_{k} \neq a_{i_{1}}+\cdots+b_{j_{k}}$ for any $k$ and index set, we can put

$$
\phi_{f}(g)=\left.g\right|_{\Lambda}\left(y_{1}, \ldots, y_{n}\right)
$$


in the diagram for any map $g \in \mathcal{E}(M(X))$.

Theorem 3.8 1. When $2<m<n$, the natural projection $p_{n, m}: U(n) \rightarrow$ $U(n) / U(m)$ is a rational $\mathcal{E}$-map if and only if $n<5$.

2. When $2<m<n$, the natural projection $p_{n, m}: S U(n) \rightarrow S U(n) / S U(m)$ is a rational $\mathcal{E}$-map if and only if $n<8$.

Lemma 3.9 Let $X=S^{a_{1}} \times \cdots \times S^{a_{m}}$ and $Y=X \times S^{b_{1}} \times \cdots \times S^{b_{n}}$ for odd-integers $a_{1} \leq \cdots \leq a_{m} \leq b_{1} \leq \cdots \leq b_{n}$. Then the first factor inclusion map $f: X \rightarrow Y$ $i$ a rational co-E-map if and only if there is no subset $\left\{i_{1}, \ldots, i_{k}\right\}$ of $\{1, \ldots, m\}$ with $b_{k}=a_{i_{1}}+\cdots+a_{i_{k}}$ for $k=1, \ldots, n$.

Proof Let $M(X)=\left(\Lambda\left(x_{1}, \ldots, x_{m}\right), 0\right)$ and $M(Y)=\left(\Lambda\left(x_{1}, \ldots, x_{m}, y_{1}, \ldots, y_{n}\right), 0\right)$ with $\left|x_{i}\right|=a_{i}$ and $\left|y_{i}\right|=b_{i}$. If $b_{k}=a_{i_{1}}+\cdots+a_{i_{k}}$, there is a map $g \in \mathcal{E}(M(Y))$ such that

$$
g\left(x_{i}\right)=x_{i} \quad(i \leq m), \quad g\left(y_{i}\right)=y_{i} \quad(i \neq k), \quad g\left(y_{k}\right)=y_{k}+x_{i_{1}} \cdots x_{i_{k}}
$$

and $M(f)\left(x_{i}\right)=x_{i}$ and $M(f)\left(y_{i}\right)=0$ for all $i$. Then we cannot have a DGAhomotopy commutative diagram

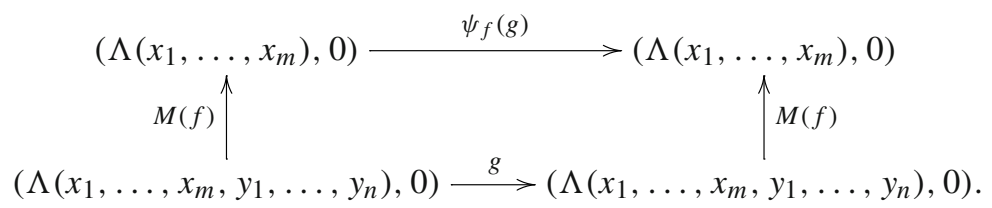

If $b_{k} \neq a_{i_{1}}+\cdots+a_{i_{k}}$ for any $k$ and $\left\{i_{1}, \cdots, i_{k}\right\}$, we can put

$$
\psi_{f}(g)=\left.g\right|_{\Lambda\left(x_{1}, \ldots, x_{m}\right)}
$$

in the diagram for any map $g \in \mathcal{E}(M(Y))$.

From Lemma 3.9, we have the following.

Theorem 3.10 1. When $2<m<n$, the natural inclusion map $i_{m, n}: U(m) \rightarrow U(n)$ is a rational co-E-map if and only if $n<5$.

2. When $2<m<n$, the natural inclusion map $i_{m, n}: S U(m) \rightarrow S U(n)$ is a rational co-E-map if and only if $n<8$.

3. When $m \leq 4$, the natural inclusion map $i_{m, n}: S p(m) \rightarrow \operatorname{Sp}(n)$ is a rational co-E-map for any $m \leq n$. When $4<m<n$, the natural inclusion map $i_{m, n}$ : $\operatorname{Sp}(m) \rightarrow \operatorname{Sp}(n)$ is a rational co-E-map if and only if $n<14$.

Proof (3) For $S=\{3,7,11,15,19,23,27,31,35,39,43,47,51,55, \ldots\}$, there are no integers $a, b, c, d \in S$ with $a<b<c<d$ satisfying the equation $a+b+c=d$ since

$$
(4 i-1)+(4 j-1)+(4 k-1)=4(i+j+k)-3 \neq 4 l-1
$$


for any $i, j, k, l \in \mathbb{N}$. On the other hand, $3+7+11+15+(19+4 i)=55+4 i=\left|v_{14+i}\right|$ for $i \geq 0$.

For a connected closed sub-Lie group $H$ of a compact connected Lie group $G$ with inclusion $j: H \rightarrow G$, there is the induced map $B j: B H \rightarrow B G$ between the classifying spaces. It induces a map $B j^{*}: M(B G)=\left(\Lambda V_{B G}, 0\right)=\left(\mathbb{Q}\left[x_{1}, \ldots, x_{k}\right], 0\right) \rightarrow$ $\left(\Lambda V_{B H}, 0\right)=M(B H)$ between the models. Here $\left|x_{i}\right|$ are even and $\operatorname{rank} G=k$. Let $V_{G}^{n}=V_{B G}^{n+1}$ by corresponding $y_{i}$ to $x_{i}$ with $\left|y_{i}\right|=\left|x_{i}\right|-1$.

Lemma 3.11 ([8, Proposition 15.16]) The (non-minimal) model of $G / H$ is given as $\left(\Lambda V_{B H} \otimes \Lambda V_{G}, d\right)$ where $d x_{i}=0$ and $d y_{i}=B j^{*}\left(x_{i}\right)$ for $i=1, \ldots, k$.

Proof of Theorem 1.4. (2) For $f: G \rightarrow G / H, M(f)$ is given by the projection $\left(\Lambda V_{B H} \otimes \Lambda V_{G}, d\right) \rightarrow\left(\Lambda V_{G}, 0\right)$ sending elements of $\Lambda V_{B H}$ to zero from Lemma 3.11. Thus we can define $\psi_{f}(g)$ for any $g \in \mathcal{E}\left(\Lambda V_{B H} \otimes \Lambda V_{G}, d\right)$ by $\psi_{f}(g)=\bar{g}$ because $g\left(x_{i}\right) \in \mathbb{Q}\left[x_{1}, \ldots, x_{k}\right]$.

Example 3.12 Let $X$ be a $G$-space for a Lie group $G$. When is the orbit map $f: X \rightarrow$ $X / G$ a rational co- $\mathcal{E}$-map ? Let $X=S^{2} \times S^{3}$, where $M\left(S^{2} \times S^{3}\right)=(\Lambda(x, y, z), d)$ with $d x=d z=0$ and $d y=x^{2}$ of $|x|=2,|y|=|z|=3$. There are free $S^{1}$-actions on $X$ where $M\left(X / S^{1}\right)=M\left(E S^{1} \times S^{1} X\right)=(\Lambda(t, x, y, z), D)$ for $M\left(B S^{1}\right)=(\mathbb{Q}[t], 0)$ with $|t|=2[1,10]$. If the Borel space of a $S^{1}$-action has the model with $D x=D t=0$, $D y=x^{2}$ and $D z=t^{2}$ (it is given by a free action on $S^{3}$ ), $f$ is not a rational co- $\mathcal{E}$ map. Indeed, we can not define $\psi_{f}(g)$ for the DGA-map $g$ with $g(x)=t, g(t)=x$, $g(y)=z$ and $g(z)=y$. But if a $S^{1}$-action has the model with $D y=x^{2}+a t^{2}$ and $D z=x t$ for $a \notin \mathbb{Q}^{*} /\left(\mathbb{Q}^{*}\right)^{2}$, the orbit map $f$ is a rational co- $\mathcal{E}$-map.

Remark 3.13 Even if a map $f$ is an $\mathcal{E}$-map, it may not be a rational $\mathcal{E}$-map. Recall an example of Arkowitz and Lupton [3]: Let $Y$ be a rational (non-universal) space such that $M(Y)=\left(\Lambda\left(x_{1}, x_{2}, y_{1}, y_{2}, y_{3}, z\right), d\right)$ with $\left|x_{1}\right|=10,\left|x_{2}\right|=12,\left|y_{1}\right|=41$, $\left|y_{2}\right|=43,\left|y_{3}\right|=45,|z|=119$,

$$
\begin{gathered}
d x_{1}=d x_{2}=0, \quad d y_{1}=x_{1}^{3} x_{2}, \quad d y_{2}=x_{1}^{2} x_{2}^{2}, \quad d y_{3}=x_{1} x_{2}^{3} \quad \text { and } \\
d z=x_{2}\left(y_{1} x_{2}-x_{1} y_{2}\right)\left(y_{2} x_{2}-x_{1} y_{3}\right)+x_{1}^{12}+x_{2}^{10} .
\end{gathered}
$$

Then $\mathcal{E}(Y)=\left\{g_{1}, g_{2}\right\}(\cong\{1,-1\})$ where $g_{1}=i d_{Y}$ and $g_{2}$ is given by

$$
\begin{gathered}
g_{2}\left(x_{1}\right)=x_{1}, \quad g_{2}\left(x_{2}\right)=-x_{2}, \quad g_{2}\left(y_{1}\right)=-y_{1}, \\
g_{2}\left(y_{2}\right)=y_{2}, \quad g_{2}\left(y_{3}\right)=-y_{3}, \quad g_{2}(z)=z
\end{gathered}
$$

[3, Example 5.2]. Consider the 12-dimensional homotopy generator $f: S^{12} \rightarrow Y$ corresponding to $x_{2}$. It is an $\mathcal{E}$-map by the homotopy commutative diagram: 


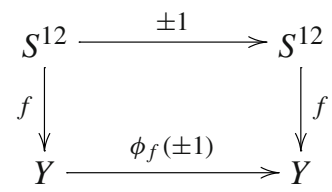

where $\phi_{f}: \mathcal{E}\left(S^{12}\right)=\{ \pm 1\} \cong \mathcal{E}(Y)$ by $\phi_{f}(1)=g_{1}$ and $\phi_{f}(-1)=g_{2}$. But it is not a rational $\mathcal{E}$-map. Because there is no map $\phi_{f}: \mathcal{E}\left(M\left(S^{12}\right)\right)=\mathcal{E}(\Lambda(u, v), d) \rightarrow$ $\mathcal{E}(M(Y))$ when $a \neq \pm 1 \in \mathbb{Q}^{*}$, i.e., there is no homotopy commutative diagram:

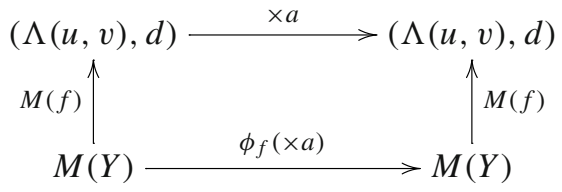

where $M(f)\left(x_{2}\right)=u, M(f)(z)=u^{8} v$ and $M(f)$ sends the others to zero. Here $M\left(S^{12}\right)=(\Lambda(u, v), d)$ with $|u|=12,|v|=23, d u=0, d v=u^{2}$ and $\times a(u)=a u$, $\times a(v)=a^{2} v$.

Proof of Theorem 1.7 1. It is obvious from the definition.

2. As (non-graded) DGAs, $M\left(\mathbb{C} P^{n}\right) \cong M\left(\mathbb{H} P^{n}\right) \cong(\Lambda(x, y), d)$ where $d x=0$ and $d y=x^{n+1}$. Therefore the inclusions $S^{2} \rightarrow \mathbb{C} P^{n}$ and $S^{4} \rightarrow \mathbb{H} P^{n}$ induce $\mathcal{E}\left(S_{\mathbb{Q}}^{2}\right) \cong \mathcal{E}\left(\mathbb{C} P_{\mathbb{Q}}^{n}\right)\left(\cong \mathbb{Q}^{*}:=\mathbb{Q}-0\right)$ and $\mathcal{E}\left(S_{\mathbb{Q}}^{4}\right) \cong \mathcal{E}\left(\mathbb{H} P_{\mathbb{Q}}^{n}\right)\left(\cong \mathbb{Q}^{*}\right)$, respectively.

3. Suppose that $m$ is even and $n$ is odd. Then $M\left(S^{m} \times S^{n}\right)=(\Lambda(x, y, z), d)$ where $d x=d y=0$ and $d z=x^{2}$ with $|x|=m,|y|=n$ and $|z|=2 m-1$.

If $m-1=n$, we have $|z|=|x y|$. Then any element of $\mathcal{E}(\Lambda(x, y, z), d)$ is given as

$$
x \rightarrow a x, \quad y \rightarrow b y, \quad z \rightarrow a^{2} z+c x y
$$

for some $a, b \in \mathbb{Q}^{*}$ and $c \in \mathbb{Q}$. The same is not true for $\mathcal{E}\left(\left(S^{m} \vee S^{n}\right) \mathbb{Q}\right)=$ $\mathcal{E}\left((\Lambda(x, y, z, \ldots), d)\right.$ since $[x y]=0$ in $H^{*}\left(S^{m} \vee S^{n} ; \mathbb{Q}\right)=\mathbb{Q}[x] \otimes \Lambda(y) /\left(x^{2}, x y\right)$, i.e, $c=0$.

If $2 m-1=n$, we have $|z|=|y|$. Then any element of $\mathcal{E}(\Lambda(x, y, z), d)$ is given as

$$
x \rightarrow a x, \quad y \rightarrow b y, \quad z \rightarrow a^{2} z+c y
$$

for some $a, b \in \mathbb{Q}^{*}$ and $c \in \mathbb{Q}$. The same is true for $\mathcal{E}\left(\left(S^{m} \vee S^{n}\right)_{\mathbb{Q}}\right)=$ $\mathcal{E}((\Lambda(x, y, z, \ldots), d)$, which is also checked by using its Quillen model $[6,8]$.

In the other case, $c=0$ for $S^{m} \vee S^{n}$ and $S^{m} \times S^{n}$.

Thus we have the following table: 


\begin{tabular}{lll}
\hline $\mathcal{E}\left(\left(S^{m} \vee S^{n}\right) \mathbb{Q}\right)$ & $\mathcal{E}\left(\left(S^{m} \times S^{n}\right) \mathbb{Q}\right)$ & $m$ :even, $n$ :odd \\
\hline $\mathbb{Q}^{*} \times \mathbb{Q}^{*}$ & $\mathbb{Q}^{*} \times \mathbb{Q}^{*} \times \mathbb{Q}$ & $m-1=n$ \\
$\mathbb{Q}^{*} \times \mathbb{Q}^{*} \times \mathbb{Q}$ & $\mathbb{Q}^{*} \times \mathbb{Q}^{*} \times \mathbb{Q}$ & $2 m-1=n$ \\
$\mathbb{Q}^{*} \times \mathbb{Q}^{*}$ & $\mathbb{Q}^{*} \times \mathbb{Q}^{*}$ & other \\
\hline
\end{tabular}

Hence the inclusion $f: S^{m} \vee S^{n} \rightarrow S^{m} \times S^{n}$ induces $\phi_{f}: \mathcal{E}\left(\left(S^{m} \vee S^{n}\right) \mathbb{Q}\right) \cong$ $\mathcal{E}\left(\left(S^{m} \times S^{n}\right)_{\mathbb{Q}}\right)$ if and only if $m \neq n+1$.

4. Since the fibrations are non-trivial, the models of the total spaces are uniquely determined as $M(E) \cong\left(\Lambda\left(x, y, v_{1}\right), d\right)$ with $|x|=m,|y|=n, d x=d y=0$ and $d v_{1}=x y$ and $M\left(E^{\prime}\right) \cong\left(\Lambda\left(x, y, v_{1}, v_{2}\right), d\right)$ with $d x=d y=0$, $d v_{1}=x y, d v_{2}=x v_{1}$. Then $\psi_{p_{\mathbb{Q}}^{\prime}} \circ \psi_{p_{\mathbb{Q}}}: \mathcal{E}(\Lambda(x, y), 0) \cong \mathcal{E}\left(\Lambda\left(x, y, v_{1}\right), d\right) \cong$ $\mathcal{E}\left(\Lambda\left(x, y, v_{1}, v_{2}\right), d\right)$ from degree reasons.

5. Recall the rigid model of Arkowitz and Lupton [3]: Let

$$
M=\left(\Lambda\left(x_{1}, x_{2}, y_{1}, y_{2}, y_{3}, z\right), d\right)
$$

with given by $\left|x_{1}\right|=8,\left|x_{2}\right|=10,\left|y_{1}\right|=33,\left|y_{2}\right|=35,\left|y_{3}\right|=37$ and $|z|=119$,

$$
\begin{gathered}
d x_{1}=d x_{2}=0, d y_{1}=x_{1}^{3} x_{2}, d y_{2}=x_{1}^{2} x_{2}^{2}, d y_{3}=x_{1} x_{2}^{3} \text { and } \\
d z=\alpha:=x_{1}^{4}\left(y_{1} x_{2}-x_{1} y_{2}\right)\left(y_{2} x_{2}-x_{1} y_{3}\right)+x_{1}^{15}+x_{2}^{12} .
\end{gathered}
$$

(Note that the degrees of elements are determined by the differential $d$ ). Then $\mathcal{E}(M)=\left\{i d_{M}\right\}$ [3, Example 5.1]. Now define a Hirsch extension [9] by $M$ as

$$
(\Lambda(v, w), 0) \rightarrow\left(\Lambda(v, w) \otimes M, d^{\prime}\right)=: M^{\prime}
$$

where $|v|$ and $|w|$ are suitable odd integers with $|v| \neq|w|,|v|+|w|=120$ and $d^{\prime} v=d^{\prime} w=0, d^{\prime} z=\alpha+v w\left(d^{\prime}=d\right.$ for the other elements). For example, put $|v|=53$ and $|w|=67$. Then any DGA-automorphism $h$ of $M^{\prime}$ is DGA-homotopic to a map with no unipotent part. Indeed, let

$$
h(z)=z+f_{1} v+f_{2} w, \quad h(v)=a v, \quad h(w)=b w+f_{3} v
$$

where $a, b \in \mathbb{Q}^{*}$ with $a b=1$ and $f_{1}, f_{2}, f_{3} \in\left(x_{1}, x_{2}\right)$, which is the ideal generated by $x_{1}, x_{2}$. Then, since $\left|f_{1}\right|=66,\left|f_{2}\right|=52$ and $\left|f_{3}\right|=14$, we have

$$
f_{1}=k_{1} x_{1}^{2} x_{2}^{5}+l_{1} x_{1}^{7} x_{2}, \quad f_{2}=k_{2} x_{1}^{4} x_{2}^{2}, \quad f_{3}=0
$$

for $k_{1}, l_{1}, k_{2} \in \mathbb{Q}$. Thus $f_{1}$ and $f_{2}$ are $d^{\prime}$-exact cocycles. Therefore $h$ is DGAhomotopic to the map with $f_{1}=f_{2}\left(=f_{3}\right)=0$ [3,9]. Hence any element $h \in$ $\mathcal{E}\left(M^{\prime}\right)$ is determined by $h(v)=a v$ and $h(w)=b w$ for $a, b \in \mathbb{Q}^{*}$ such that $a b=1$ (since $\left.\left.h\right|_{M} \sim i d_{M}\right)$. Thus we obtain

$$
\mathcal{E}\left(M^{\prime}\right)=\left\{(a, b) \in \mathbb{Q}^{*} \times \mathbb{Q}^{*} \mid a b=1\right\} \cong \mathbb{Q}^{*} .
$$


Therefore the DGA-surjections $(\Lambda v, 0) \stackrel{f^{*}}{\leftarrow} M^{\prime} \stackrel{g^{*}}{\rightarrow}(\Lambda w, 0)$ (spherically injective maps $f: S_{\mathbb{Q}}^{|v|} \rightarrow\left\|M^{\prime}\right\|$ and $g: S_{\mathbb{Q}}^{|w|} \rightarrow\left\|M^{\prime}\right\|$, which are their geometric realizations) with $f^{*}(M)=g^{*}(M)=0, f^{*}(v)=v, g^{*}(w)=w$ and $f^{*}(w)=$ $g^{*}(v)=0$ induce

$$
\left(\mathbb{Q}^{*} \cong\right) \mathcal{E}\left(S_{\mathbb{Q}}^{|v|}\right)=\mathcal{E}(\Lambda v, 0) \underset{\overline{\phi_{f}}}{\cong} \mathcal{E}\left(M^{\prime}\right) \underset{\psi_{g}}{\cong} \mathcal{E}(\Lambda w, 0)=\mathcal{E}\left(S_{\mathbb{Q}}^{|w|}\right)
$$

with $\psi_{g} \phi_{f}(a)=a^{-1}$ for $a \in \mathbb{Q}^{*}$. Thus we have $S_{\mathbb{Q}}^{|v|} \widetilde{\mathcal{E}} S_{\mathbb{Q}}^{|w|}$.

Acknowledgements The authors are grateful to Antonio Viruel for his interest and suggesting examples in [7] and Jim Stasheff for his kind support.

\section{References}

1. Allday, C., Puppe, V.: Cohomological Methods in Transformation Groups, Vol. 32. Cambridge Studies in Advanced Mathematics (1993)

2. Arkowitz, M., Curjel, C.R.: Groups of Homotopy Classes. Lecture Notes in Math, vol. 4. Springer, Berlin (1967)

3. Arkowitz, M., Lupton, G.: Rational obstruction theory and rational homotopy sets. Math. Z. 235(3), 525-539 (2000)

4. Benkhalifa, M.: Rational self-homotopy equivalences and Whitehead exact sequence. J. Homotopy Relat. Struct. 4(1), 111-121 (2009)

5. Benkhalifa, M.: Realizability of the group of rational self-homotopy equivalences. J. Homotopy Relat. Struct. 5(1), 361-372 (2010)

6. Benkhalifa, M., Smith, S.B.: The effect of cell-attachment on the group of self-equivalences of an R-localized space. J. Homotopy Relat. Struct. 10(3), 549-564 (2015)

7. Costoya, C., Viruel, A.: Every finite group is the group of self homotopy equivalences of an elliptic space. Acta Math. 213, 49-62 (2014)

8. Félix, Y., Halperin, S., Thomas, J.C.: Rational Homotopy Theory. Graduate Texts in Math, Vol. 205, Springer, New York (2001)

9. Griffiths, P., Morgan, J.: Rational Homotopy Theory and Differential Forms. Birkhäuser, Basel (1981)

10. Halperin, S.: Rational Homotopy and Torus Actions. London Math. Soc. Lecture Note Series, Cambridge University Press, Cambridge 93, 293-306 (1985)

11. Hilton, P.: Homotopy Theory and Duality. Gordon and Breach Science Publishers, New York (1965)

12. Hilton, P., Mislin, G., Roitberg, J.: Localization of nilpotent groups and spaces. North-Holland Math. Studies 15 (1975)

13. Lee, K.: The groups of self pair homotopy equivalences. J. Korean Math. Soc. 43(3), 491-506 (2006)

14. Kim, J., Oda, N.: The set of cyclic-element preserving maps. Topol. Appl. 160(6), 794-805 (2013)

15. Maruyama, K., Rutter, J.: Groups of homotopy self-equivalences and related topics. Contemp. Math. 274, American Math Soc (1999)

16. Piccinini, R.A.: Groups of Self-equivalences and Related Topics. Lecture Notes in Math, vol. 1425. Springer, Berlin (1990)

17. Rutter, J.W.: Spaces of Homotopy Self-equivalences A Survey. Lecture Notes in Math, vol. 1662. Springer, Berlin (1997)

18. Sullivan, D.: Infinitesimal computations in topology. Inst. Hautes Études Sci. Publ. Math. 47(47), 269-331 (1977)

19. Toda, H.: Composition Methods in Homotopy Groups of Spheres, Vol. 49. Annals of Mathematics Studies, Princeton University Press, Princeton, NJ (1962)

20. Vigué-Poirrier, M., Sullivan, D.: The homology theory of the closed geodesic problem. J. Differ. Geom. 11(4), 633-644 (1976) 